\title{
Identification and antimicrobial susceptibility profile of bacterial pathogens isolated from wound infections in a tertiary hospital, Bayelsa South southern, Nigeria
}

\author{
Abdu A.B. ${ }^{1}$, Egbagba J. ${ }^{2}$, Fente B.G. ${ }^{3}$ \\ ${ }^{1}$ Abdulrashed B. Abdu, Department of Medical Microbiology and Parasitology, Faculty of Basic Medical Sciences, College of \\ Health Sciences, Niger Delta University, Wilberforce Island, Amassoma, Bayelsa-State, ${ }^{2}$ John Egbagba, Department of Medical \\ Microbiology and Parasitology, Federal Medical Centre, Yenagoa, Bayelsa-State, ${ }^{3}$ Beleudanyo G. Fente, Department of Surgery, \\ Faculty of Clinical Sciences, College of Health Sciences, Niger Delta University, Wilberforce Island, Amassoma, Bayelsa-State, \\ Nigeria.
}

Corresponding Author: Abdulrashed B. Abdu, Department of Medical Microbiology and Parasitology, Faculty of Basic Medical Sciences, College of Health Sciences, Niger Delta University, Wilberforce Island, Amassoma, Bayelsa-State, Nigeria E-mail: abdulsoul@gmail.com

\begin{abstract}
Introduction: Infected wounds in particular, those associated with antimicrobial resistant microorganisms constitute a significant source of physical, psychological, and economic burden to patients in particular, and the society at large in terms of the huge resources spent in treating such wounds as well as time/days lost at workplaces leading to increased morbidity and mortality globally. The present study sought to investigate and identify the common bacteria associated with wound infections and their antibiotic sensitivity pattern. Material and Methods: A total of 130 wound samples were collected by doctors in ward using Sterile Swab Sticks over a period of 6 months from February-July 2019. Pathogenic bacteria were isolated, identified and their antibiotic susceptibility tests were performed. Results: Among 130 cases, 108 (83.08\%) were culture positive for bacterial pathogens, while $22(16.92 \%)$ were bacteriologically sterile (Negative). Rate of infection was high in males $(56.15 \%)$ than females (43.85\%). Of the 108- culture positive, 164 isolates were recovered of which 28 (17.07) were anaerobes, while 136 were aerobes of which 106 (64.64\%) were Gram-negative species and 30 (18.29\%) Gram-negative species. The predominant isolates among the facultative aerobes, were Pseudomonas aeruginosa 28 (17.07\%), followed by E. coli 19 (11.58\%), Klebsiella pneumonia 17 (10.37\%) while the least were amongst Morganella morgani, Providencia rettgeri and Serretia marcescens with $2(1.22 \%)$ individually. Among the Gram-positive, Staphylococcus aureus $10(6.10 \%)$ were most common, followed by Enterococcus spp 4 (2.44\%), Coagulase negative staphylococcus (CoNS) 4 (2.44\%) while the least were amongst Streptococcus Group B, Streptococcus Group C, Streptococcus Group G with 2 (1.22\%) separately. Amongst the anaerobes, Bacterioides fragilis 16 (9.75\%) is the most common, followed by Peptostreptococcus spp., and Prevotella spp with 4 (2.44\%) each, while Fusobacterium spp and Veillonella spp were the least, 2 (1.22\%) each. Amikacin and nitrofurantoin are the most efficacious for managing wound infections, with all the isolates being susceptible to them. Resistance was moderate to Levofloxacin (49.39\%). On the other hand, resistance were above average among Ciprofloxacin (96.34\%), Cloxacillin (94.51\%), Ceftriaxone (91.46\%), Ceftazidine (91.46\%), Cefotaxime (87.20\%), Erythromycin (59.15\%), Ofloxacin(58.54\%) and Chloramphenicol (51.22\%). The least resistance shown to Gentamicin (16.46\%), Streptomycin (16.46\%), Imipenem (7.93\%), Metronidazole (7.93\%), and Piperacillin-tazobactam (7.93\%). Conclusion: Antibiotic susceptibility test is suggested prior to administration of antibiotics for successful treatment and intermittent monitoring is also encouraged to check emerging multidrug resistant trends as a guide to health authorities.
\end{abstract}

Keywords: Anaerobic, Wound infections, Antibiotic resistance, Swab sample

\section{Introduction}

Wound is termed as an injury to any of the body tissues specifically when it is caused by physical means that

Manuscript received: $15^{\text {th }}$ October 2019

Reviewed: $25^{\text {th }}$ October 2019

Author Corrected: $1^{\text {st }}$ November 2019

Accepted for Publication: $5^{\text {th }}$ November 2019 interrupts continuity of epithelium, with or without loss of underlying connective tissue [1]. Wounds can be categorised as accidental, pathological or post-operative [2] and it provides a moist, warm, and nutritious environment that is favourable to microbial adherence, colonisation and proliferation causing impairment to the host tissue [3].

Pathology Update: Tropical Journal of Pathology \& Microbiology Available online at: www.medresearch.in 966 | P a g e 
Different species of bacteria are normal flora on human skin in the nasopharnyx, gastrointestinal tract and other parts of the body with little potential to cause disease, because of the first line of defense of the body. Despite the skin barrier, any breach in the skin surface whether trauma, accident, surgical operation or burn will open the door for bacterial infections [4].

Since wound colonisation is most frequently polymicrobial, involving varied collection of microorganisms that are potentially pathogenic, any wound is at some risk of becoming infected [5], even though, majority of the wounds may not get infected [6].

Infection in wound creates a major impede to healing and can have an unpleasant impact on the patient's value of life as well as on the healing rate of the wound [7]. Infected wounds are likely to be more painful, hypersensitive and odorous, resulting in amplified uneasiness and discomfort for the patient [8].

The introduction of antimicrobial therapy in the management of wound infections has reduced the treatment cost; long duration of hospital stays and significant morbidity and mortality arising from infective wounds, however, the development of resistance to these antibiotics presents a challenge to the efficient management of wound infection [9]. There has been a rise of antibiotic resistance in hospitals and communities which has been associated with the use of antibiotics [10]. Regrettably, the increased costs of screening for effective antimicrobial agents and the declined rate of newer innovative drugs discovery has made the situation increasingly bothersome [11].

Very few studies of this kind have been carried out in Bayelsa, and this issue was largely unexplored in FMC, Yenagoa. However, in previous study conducted by Pondei et al [12], in Niger Delta University Teaching Hospital, Okolobiri (a tertiary hospital), 87 (86.13\%) bacteria were isolated from wound specimens. They also evaluate the antimicrobial susceptibility profiles of the bacteria isolates so as to assess the effectiveness in vitro of the conventional antibiotics that will be used to treat bacterial infections in wound patients. There is a need for a more rational approach to the use of antibiotics based on microbial prevalence and antibiotic susceptibility in the management of infected wounds, for the knowledge of the causative agents of wound infection has proved to be helpful in the selection of empiric antimicrobial therapy and on infection control measures in hospitals [13]. Furthermore, they are also useful in formulating rational antibiotic policies. Thus, it is necessary for us in the present study to determine the pattern of microbiological profile of wounds and the current susceptibility profile of the microbiological organisms isolated from such in our setting which will serve as a guide for empirical antibiotic use.

\section{Materials and Methods}

Study design- This descriptive cross-sectional study of isolates from the wounds of patients at Federal Medical Centre, Yenagoa, Bayelsa-State from February to July 2019.

Study area- Federal Medical Centre (FMC), Yenagoa, is one of the tertiary medical institution in Yenagoa, southsouthern region catering to about 1,704,515 inhabitants of Bayelsa, and neighboring states of Delta and Rivers

Inclusion/exclusion criteria- All patients attending outpatient and those admitted to surgery ward with suspected wound infections were enrolled in the study. Wound infection was suspected if the wound was exuding pus or fluid, not healing well or associated with signs and symptoms of inflammation. Very ill patients and those with clinical or histopathological evidence of malignancy were excluded from the study. Patient's age, sex, type, site, duration and aetiology of the wound were noted.

Ethical committee clearance for the study was granted by the Ethics Review Board of Federal Medical Centre, Yenagoa.

Sampling procedure- The patients were enrolled sequentially, and informed consent was obtained from all assenting patients. A questionnaire was used to obtain data from the patients. Sample collection was performed by the surgeon by obtaining a wound swab from each patient by employing the classic Levine technique as described by Smith et al. [14] after cleaning the wound surface with saline using sterile swab sticks. Only one swab per patient was collected after carefully cleaning the wound and surrounding skin with saline to avoid surface contamination. The samples were then transferred to microbiology laboratory within $1 \mathrm{~h}$ of collection using airtight sterile vial.

Culture and identification- In the laboratory, swabs collected were streaked on blood agar, Chocolate agar, and MacConkey agar (Oxoid, England) by sterile inoculation loop. Agar plates were incubated aerobically and anaerobically (anaerobic jar at $37^{\circ} \mathrm{C}$ for $24-48 \mathrm{~h}$ ). Bacterial colonies on the agar plates were identified using routine clinical microbiology test procedures, including Gram stain, catalase, coagulase, oxidase, and indole.

Colonies with Gram-positive coccal appearance on Gram staining were tested for the enzyme catalase to distinguish Staphylococci from Streptococci. Staphylococci were 
tested for coagulase production by the slide tests using citrated human plasma. Confirmation of Gram-positive isolates were done on Staph identification 25E (BioMeriux, Marcy l'Etoile, France), while colonies with Gram-negative bacilli appearance on Gram staining were subjected to Oxidase test to differentiate the Enterobacteriaceae (oxidase-negative) from other Gram-negative bacteria (oxidase-positive). Gram-negative bacilli identification was confirmed with the use of API 24E and API 20NE, while the anaerobes were confirmed with ID 32A (Biomerieux, France) identification test kit.

Antibiotic sensitivity- Antibiotic susceptibility was done by Kirby-Bauer disc diffusion method and interpreted according to the recommendations of the National Committee for Clinical Laboratory Standards (CLSI, 2019) [15]. The drugs tested for the Anaerobes, Gram-positive and Gram-negative bacteria were amikacin $(10 \mu \mathrm{g})$, ampicillin $(25 \mu \mathrm{g})$, amoxicillin-clavulanic acid $(30 \mu \mathrm{g})$, cefotaxime $(30 \mu \mathrm{g})$, ceftriaxone $(30 \mu \mathrm{g})$, ceftazidime $(30 \mu \mathrm{g})$, chloramphenicol $(30 \mu \mathrm{g})$, ciprofloxacin $(5 \mu \mathrm{g})$, cloxacillin $(5 \mu \mathrm{g})$, erythromycin $(15 \mu \mathrm{g})$, gentamicin $(10 \mu \mathrm{g})$, imipenem $(10 \mu \mathrm{g})$, levofloxacin $(5 \mu \mathrm{g})$, metronidazole $(50 \mu \mathrm{g})$, nitrofurantoin $(300 \mu \mathrm{g})$, ofloxacin $(5 \mu \mathrm{g})$, piperacillintazobactam $(110 \mu \mathrm{g})$, streptomycin $(10 \mu \mathrm{g})$, and tetracycline $(30 \mu \mathrm{g})$. All the discs were obtained from Oxoid, England. And the antibiotics selected were based on the availability and prescription frequency of these drugs in the study area.

Statistical analysis- The data generated from each patient was entered into the Statistical Package for the Social Science (SPSS) version 20 (Chicago, SPSS Inc) sheet and analysed with the appropriate test statistics. $\mathrm{P}<0.05$ was considered statistically significant.

\section{Results}

A total of 130 (80 [61.54\%] acute; 50 [38.46\%] chronic) wound swab samples were collected. The subjects included 73 (56.15\%) males and 57 (43.85\%) females (ratio 1.3:1). The ages of the patients ranged from 6 months to 70 years with a mean age of 34.5 years. Seventy-three patients $(56.15 \%)$ were managed as inpatients while, 57 (43.85\%) were outpatients. Posttraumatic wounds were the most frequent wounds sampled accounting for $54(41.78 \%)$ of all the wounds; other aetiologies are represented in Figure 1.

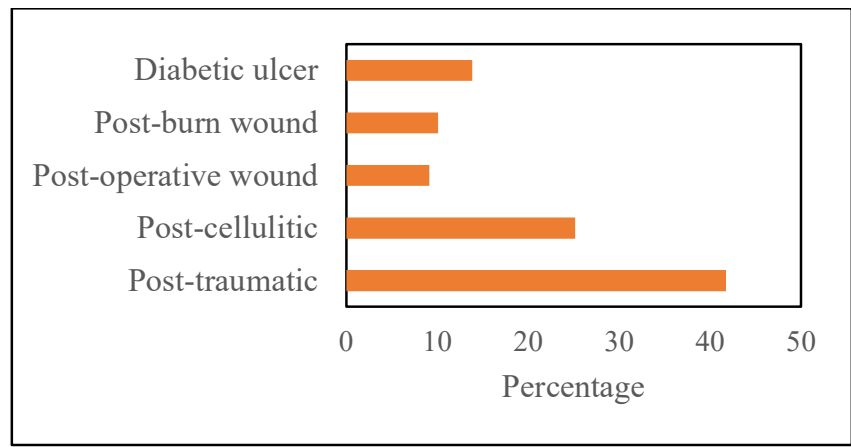

Fig-1: Wound type distribution

From these 130 cultures, 108 (83.08\%) swab samples yielded growth of microorganisms, of which 83 (76.85\%) samples grew single bacterial isolate, while $25(23.15 \%)$ samples yielded polymicrobial growth. Twenty-two (16.92\%) did not yield any growth. Of the 108-culture positive, 164 microorganisms in total were isolated, including 28 (17.07) anaerobic species, 30 (18.29\%) Gram-positive species and 106 (64.64\%) Gram-negative species (Figure 2).

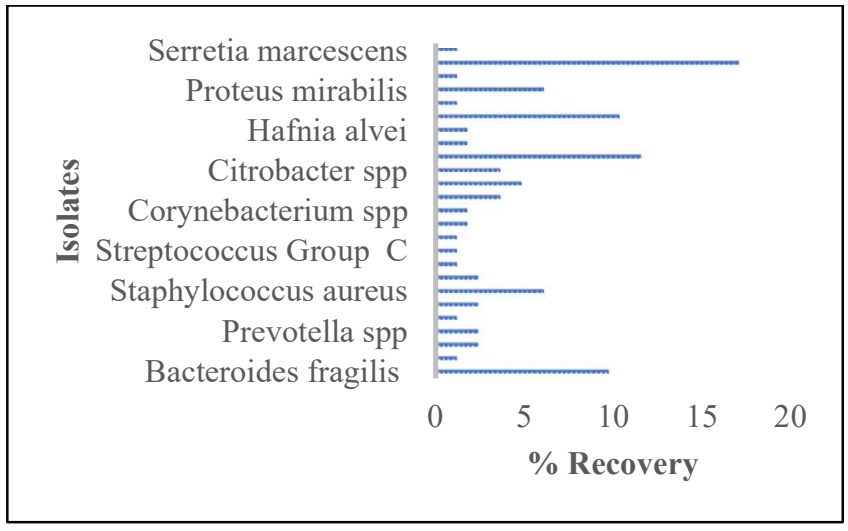

Fig-2: Frequency of bacterial isolates from the wound swabs.

Pathology Update: Tropical Journal of Pathology \& Microbiology Available online at: www.medresearch.in 968 | P a g e 
The most common organism isolated among the Gram-negative was Pseudomonas aeruginosa, which accounted for 28 (17.07\%) of all the bacterial isolates; this was followed by Escherichia coli 19 (11.58\%), while the least was Providencia rettgeri and Serretia marcescens with $2(1.22 \%)$ isolates each. The most common isolate among the Gram-positive bacterial was Staphylococcus aureus 10 (6.01\%); this is followed by the Enterococcus and Coagulase negative staphylococcus (CoNS) with $4(2.44 \%)$ isolates each, while the least were Streptococcus Group B, C and G with $2(1.22 \%)$ respectively. The most common isolates among the anaerobic bacteria was Bacteroides fragilis with16 (9.75\%) isolates, this is followed by Peptostreptococcus spp and Prevotella spp with $4(2.44 \%)$ each. The least isolate among the anaerobic bacterial was Fusobacterium spp and Veillonella spp with 2 (2.44\%) isolates each. Figure 2 shows the various isolates as obtained from the wound swabs.

All the bacterial isolates were investigated for their antimicrobial properties against 8 classes (comprising of 19) antibiotics and the antibiotic susceptibility pattern is represented in Figure 3. As shown, varying susceptibility and multidrug resistance patterns were noted among the different classes. Also, this figure shows the percentage resistance observed for each bacteria group against an individual antibiotic. Resistance percentages of $100 \%$ were noted amongst all the isolates against the antibiotic ampicillin, amoxicillin-clavulanic acid and tetracycline, while none of the isolates exhibited resistance $(0.00 \%)$ against amikacin and nitrofurantoin. The highest resistance was observed against $\beta$-lactams and tetracycline in all groups. The overall susceptibility patterns of each individual antibiotic against all the bacteria across the different groups (i.e. anaerobes, Grampositive cocci and Gram-negative bacilli) revealed that the bacteria conferred resistance to ampicillin (100\%), amoxicillinclavulanic acid $(100 \%)$, tetracycline $(100 \%)$. Above $50 \%$ of the isolates displayed resistance to ciprofloxacin $(96.34 \%)$, cloxacillin (94.51\%), cefotaxime (91.46\%), ceftriaxone (91.46\%), ceftazidime (87.20\%), erythromycin (59.15\%), ofloxacin (58.545) and chloramphenicol (51.22\%). Resistance to levofloxacin, gentamicin, streptomycin, imipenem, metronidazole and piperacillin-tazobactam were $49.39 \%, 19.85 \%, 19.85 \%, 7.93 \%, 7.93 \%$ and $7.93 \%$ respectively.

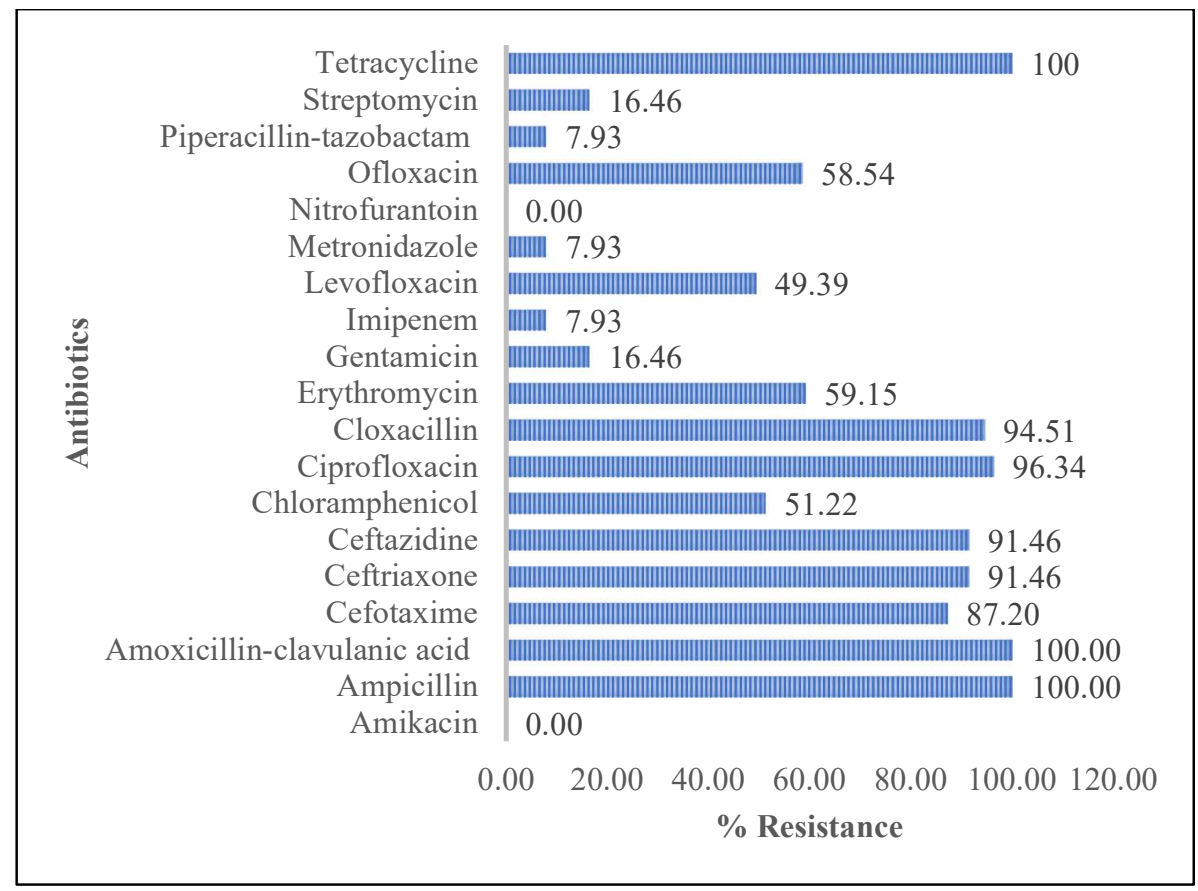

Fig-3: Susceptibility patterns of bacterial isolates cultured to various antibiotics.

\section{Discussion}

The present study investigated the prevalence of microbes attributed to wound infection in FMC, Yenagoa, because the outcome will help to diagnose the microbial pathotypes associated with our wound infections and will also guide us on antimicrobial selection, if treatment is considered necessary. For decades, debate has been ongoing on the contribution and consequence of microorganisms in wound healing. While some authorities deemed the microbial density to be dire in predicting wound healing and infection, others consider the types of microorganisms to be of greater significance. However, these and other factors which includes microbial synergy, the immune response of the host, and the quality of tissue involved must be collectively put into consideration in assessing the possibility of infection. In addition, discussion is still ongoing regarding the value of wound sampling, the types of wounds that should be sampled, and the sampling technique required to yield the most meaningful data. In the laboratory, consideration must be given to the relevance of culturing polymicrobial specimens, the value in identifying one or 
more microorganisms, and the microorganisms that should be assayed for antibiotic susceptibility. The present study employed the less invasive (swab culture) techniques for collection of wound specimens, because this method as ascribed by Smith et al [14] and Angel et al [16], is considered less invasive than tissue biopsy or curetted tissue and are often an ideal process for culturing wounds provided the wound is cleansed and debrided prior to sampling $[14,16]$.

In the present study, rate of recovery of bacterial population from wound infection was $83.08 \%$, that is similar to other studies conducted earlier $[2,9,12,13]$, and the frequency of wound infection was slightly more common in males $(56.15 \%)$ than in females $(43.85 \%)$. This is in support with studies done earlier in a tertiary hospital (Niger Delta University Teaching Hospital, Okolobiri) situated in a semi-urban area of the Yenagoa [12], in different parts of Nigeria $[17,18]$ and in Nagpur, India [2]. Conventionally, this might be supported by the fact that males are dominants in occupations where trauma is regular, namely, commercial (tricycle) riders, industry and farming. This also relates to the fact that majority of the infected wounds were seen in the working age (mean age) group of 34.5 years and that the most common wound type was post-traumatic $(41.78 \%)$. Evidence have shown that microbial populations that colonised infected wounds reflects the diversity of the patient population polls, underlying wound aetiology, duration of treatment, technique used to collect specimens, and species $[19,20]$.

The microflora of wound is usually polymicrobial, complex and has been found in vivo to range from 1.6 to 4.4 species with the opportunity to transform over time [21]. In this present study, it was recovered both aerobic and anaerobic bacteria pathogens with the aerobic bacteria been the most common bacteria with $136(82.94 \%)$ isolates while the anaerobes represent 28 (17.07\%). On gram stain examination, $30(18.30 \%)$ pathogens were aerobic gram positive and $106(64.64 \%)$ were aerobic gram negative. This is in comparison with $31.15 \%$ Gram-positive and $68.85 \%$ gram-negative reported by Goswami et al [22] and Pondei et al [12] with $14.94 \%$ Gram-positive and $85.06 \%$ Gram negative as against $69 \%$ for gram positive and $29 \%$ for gram negative organisms as reported by Surucuoglu et al [23] in their setup. This disparity may be due to divergence in common nosocomial pathogens inhabitant in different individual environment and possibly within the hospital set up. In the current study, generally, Pseudomonas aeruginosa was the most common isolated bacteria, this is followed by E. coli, Klebsiella pneumoniae, Proteus mirabilis and Staphylococcus aureus. This observation is in dissimilarity to preceding studies, that indicted Staphylococcus aureus $[22,24]$ and E. coli $[25,26]$ as the most commonly isolated, occurring in frequencies ranging from $26.19 \%$ to $88 \%$. Other aerobic species isolated from the infected wounds in the present study as presented in descending order includes: Gram-negative bacilli: Alcaligens spp., Acinetobacter junil, Citrobacter spp., Enterobacter cloacae, Hafnia alvei, Morganella morgani, Providencia rettgeri and Serretia marcescens, while the Gram positive includes: Enterococcus spp., Coagulase negative staphylococcus, Streptococcus pneumoniae, Corynebacterium spp., and Streptococci B, C and $\mathrm{G}$ making this list widespread and multifaceted. When carrying out investigations on wound infection, where feasible, it is advised to investigate the contributions of anaerobic organisms because, wound infection due to anaerobic organisms are also frequently identified, and their contributions has been reported to be ranging in prevalence, in particular, chronic wounds from as low as $6 \%$ to as high as $88 \%$ and the culpable anaerobes include Bacteroides, Peptostreptococcus and Prevotella [27,28].

With this in mind and in that majority of investigations in our environment do not carry out studies on the contributions of anaerobes to wound infections, the present study sorted to investigate the involvements of anaerobic bacteria pathotypes in our hospital setup. The present findings revealed that anaerobic bacteria contributed $17.07 \%$ prevalence in wounds infection. This is awesome, as this report will be a point of reference in the study environment as most studies are restricted to culturing aerobic bacteria only $[9,12]$. In the present study, the isolation of anaerobes, particularly Bacteroides fragilis which is the most frequently isolates accounting for 16 (9.75\%), this is followed by Peptostreptococcus and Prevotella spps., with recovery rate of $4(2.44 \%)$ each, while the least anaerobic isolates are Fusobacterium spp., and Veillonella spp with $2(1.22 \%)$ recovery rate each. The recovery of these anaerobes from the present study indicates that anaerobes also play an influential role in wound infections in our set up, thus substantiating earlier studies [27-31].

The inferences of this outcome are that treatment of only the facultative bacteria, without adequate antibiotic coverage for anaerobic bacteria, could lead to clinical failures associated with complications of such wounds. The present study, therefore affirmed the suggestions made by Izadpanah \& Khalili [32], that the best therapeutic results from wound infections could be appreciated with antimicrobial drugs that are active against both types of microorganisms. Such therapeutic adversities have been witnessed in the treatment of mixed infections with cephalosporins and penicillins that lack significant activity against anaerobes. Similarly, for example, the use of metronidazole or clindamycin as a single agent is associated with failures caused by infection with facultative bacteria. In other words, mixed infections involve complex 
interactions between facultative bacteria and strict anaerobes, many of which maintain intrinsic pathogenicity [33]. Based on these facts, several evidences and reports including ours encourages the use of combination therapy regimens as against monotherapy for treatment of wound infections [33-36].

The choice of an effective antimicrobial agent for a microbial infection requires information of the potential microbial pathogens, an understanding of the pathophysiology of the infectious and pharmacokinetics of the proposed therapeutic agents. Hence the treatment of infections in patients becomes difficult. Studies are required to assess the right kind of antibiotics and the appropriate concentrations to be used in infections taking into consideration the aetiology of the infection and duration of the antibiotic treatment [37]. Hereafter, antibiotics are often prescribed for the supportive treatment of such wounds. The choice of antibiotic is usually based on previously available susceptibility testing and previous clinical efficiency. There is concern that bacteria have increased resistant to the currently prescribed antibiotics [38].

Susceptibility testing is the determination of the bacterial pattern of resistant to a number of antibiotics. As such the present study carried out an indebt study into the susceptibility patterns of the isolated pathogens and the outcome is quite worrying with the presence of multi-drug resistant pathogens in infected wounds of patients attending FMC, Yenagoa, as a result presenting a major risk. It is interesting though, that, despite the wide spread of resistance noticed amongst our isolates, they were all susceptible to amikacin and nitrofurantoin which is similar to the result documented previously [39-42], and in contrast to report by Pondei et al [12].

This could be explained by the rare use of aminoglycoside in our setting that is categorised as a low and middleincome country, while resistance to nitrofurantoin remains relatively rare despite several decades of widespread use because of its several mechanisms of action and its severe reaction which includes: sudden onset of fever, chills, myalgia, dyspnea, and persistent dry cough [43,44], thus restraining self-medication and indiscriminate abuse of the drug has done to other orally administered antibiotics. In addition, our isolates showed high susceptibility to other aminoglycosides (gentamicin and streptomycin).

As revealed, all the anaerobic bacteria isolates were susceptible to all aminoglycosides (amikacin (100\%), gentamicin $(100 \%)$ and streptomycin $(100 \%)$ tested. Even though studies have discouraged the use of aminoglycosides alone for managing anaerobes, because it is believed that aminoglycosides is oxygen dependent active transport, a required step making aminoglycosides ineffective against anaerobic bacteria $[45,46]$, however, the present study subjected the isolates to test in order to highlights the activities and since studies have recommend that it should be given as combinatory therapy. For example, the treatment of infections caused by anaerobic bacteria (or a mixed infection having an anaerobic component) are suggested to be used in the combinations as following: penicillins (ampicillin/ticarcillin), beta-lactambeta-lactamase inhibitor combinations (amoxicillin/ clavulanic acid, ticarcillin/clavulanic acid, ampicillin/ sulbactam, piperacillin/ tazobactam), cephalosporins (Cefoxitin, cefotetan "cephamycins"), aminoglycosides (amikacin, gentamicin, streptomycin, tobramycin), polymyxins (polymyxin B, polymyxin E, colistin methate sulfate (CMS), carbapenems (imipenem, meropenem, doripenem), clindamycin, moxifloxacin, macrolides (azithromycin, clarithromycin), quinolones (ciprofloxacin, levofloxacin), fosfomycin, rifampin, chloramphenicol, tetracyclines (minocycline and doxycycline), glycylcycline (tigecycline) and metronidazole [31,47-49].

This outcome thus supports the suggestion that aminoglycosides should be in conjoined as a combinatory antibiotic for wound management. In spite of this impressive outcome reported among the anaerobes, the findings of this study indicate existence of resistant bacteria among the isolates from wound infections within the grampositive and gram-negative facultative aerobes. For example, The Gram-negative isolates were fairly resistant to aminoglycosides: gentamicin and streptomycin with $10.36 \%$ each, while $6.10 \%$ of Gram-positive isolates were resistant to gentamicin and streptomycin individually. This pattern of antibiotic sensitivity correlates with the previous study [12].

However, contrary to our findings, many authors have reported higher level of resistance among infected wound isolates to aminoglycosides [2,22,24,50,51]. Aside from haphazard use of these drugs in those set ups, bacteria are able to resist the action of aminoglycosides via: i). Enzymatic modification (phosphorylation, acetylation \& adenylation of aminoglycodsides); ii). Plasmid mediated decreased drug uptake (efflux pumps), and iii). Target site modification (16S rRNA methylation decreases binding affinity) [45].

The present study had shown reduced resistance to carbapenems (Imipenem (7.93\%)), Metronidazole (7.93\%) and beta-lactam-beta-lactamase inhibitor combinations (piperacillin/tazobactam (7.93\%). Of the anaerobes, only one $B$. fragilis isolates showed low resistant to both imipenem and metronidazole $1(0.61 \%)$, while $3(1.83 \%)$ showed resistance to piperacillin/tazobactam. This outcome is in sharp contrast to previous study [52] but is in consistent with the observation made by Boyanova et al [53] in a study

Pathology Update: Tropical Journal of Pathology \& Microbiology Available online at: www.medresearch.in 971 | P a g e 
made in the USA for metronidazole $(<1 \%)$ and carbapenems (1\%) from 2008 to 2013 . On the other hand, all other anaerobes in the present study were susceptible to imipenem, metronidazole and piperacillin/tazobactam. The aggregate resistance rates of $4(2.44 \%)$ was observed among the gram-positive aerobes to imipenem, metronidazole and piperacillin/tazobactam. Of note, only one $(0.61 \%)$ isolate each for $S$. aureus, CoNS, $S$. pneumoniae and Corynebacterium spp. was involved. This low resistance rate among gram-positive isolates from infected wound is in consistent with earlier reports documented for imipenem [2,9] and metronidazole [2].

The current study showed that the gram-negative isolates were sensitive to imipenem, metronidazole and Pipearcillin/Tazobactam and are highly resistant to cephalosporins which makes this outcome similar to the previous reports $[25,54]$. In the present study, all the gramnegative isolates were resistance to the cephalosporins (Cefotaxime, Ceftriaxone and Cetazidine). This high resistance rate is similar to earlier report made by Pondei et al [12] in Okolobiri. Of interest is that 27 (96.43\%) of the 28 Pseudomonas showed high susceptibility towards penicillin derivatives (Pipracillin/Tazobactum) which corroborates already reported study conducted in western Nigeria [17], despite that 11 (39.29\%) of this organism showed resistant to the aminoglycosides (Gentamycin and Streptomycin). Thus, the present study suggested the inclusion of Pipracillin/Tazobactum into the management of Pseudomonas identified wound infection therapy.

In this current study, the combination of antibiotics such as carbapenems (imipenem), metronidazole, nitrofurantoin, pipracillin/tazobactum and aminoglycosides (amikacin, gentamicin, and streptomycin) were the most efficacious and could be useful combinatory empirical therapy while awaiting laboratory report. Our findings indicate the existence of high drug resistant bacteria in wound infections. For instance, all $(100 \%)$ the bacterial isolates from the infected wounds in this present study were resistant to ampicillin, amoxicillin-clavulanic acid and tetracycline. Similarly, high percentages of resistance to ampicillin and other penicillin derivatives has been recorded $[2,9,12,25,55]$.

The high use of $\beta$-lactam antibiotics and inappropriate infection control procedures in the hospitals might be attributed to the cause of rising rates of resistance among these bacteria. Moreover, longer duration of preventive antimicrobial exposure in medical interventions may contribute to organisms for developing resistance. Aside from the $\beta$-lactam that our isolates showed high degree of resistance to, the high degree of resistances were also shown to the quinolones (ciprofloxacin: $96.34 \%$, ofloxacin: 58.54\%, levofloxacin: 49.39\%), cloxacillin (94.51\%) and chloramphenicol (51.22\%). Making these drug ineffective in the management of infected wounds in our set up. This high level of resistance may be attributed to the widespread abuse of these antibiotics, practicing self-medication, indiscriminate use of antibiotics as oral prophylaxis, lack of laboratory services and guidelines/ protocols regarding the selection of antibiotics.

Study limitations- The limitations of this study include the small sample size and absence of cefoxitin discs which prevented us from determining if the isolated $S$ aureus were methicillin resistance strains despite, they been multidrug resistant.

\section{Conclusion}

From this study, anaerobic microorganisms are significant pathogens in infected wounds as such, the proper diagnosis and treatment of these infections are important healthcare priorities. The most common isolate in wound infection was Pseudomonas aeruginosa, Escherichia coli, Klebsiella pneumonia, Bacteroides fragilis, Staphylococcus aureus, Proteus mirabilis, Alcaligens spp., Acinetobacter junii, Citrobacter spp., Peptostreptococcus spp., Prevotella spp., Enterococcus spp., Coagulase negative Staphylococcus, Streptococcus pneumoniae, Corynebacterium spp., Enterobacter cloacae, Hafnia alvei, Fusobacterium spp., Veillonella spp., Streptococcus Group B, Streptococcus Group C, Streptococcus Group G, Morganella morgani, Providencia rettgeri and Serretia marcescens.

\section{What the study adds to the existing knowledge?}

These isolates showed a high degree of resistance to Ampicillin, Amoxicillin-clavulanic acid, Tetracycline, Ciprofloxacin, Cloxacillin, Ceftriaxone, Ceftazidime, Cefotaxime, Erythromycin, Ofloxacin and Chloramphenicol. There is crucial need to curb further spread of antimicrobial resistance, and the present study recommends multidisciplinary approach to wound management, rational drug use, routine microbiological surveillance of wounds and rigorous infection control policies.

\section{Author's contribution}

Abdulrashed B. Abdu: Designed the study, protocol writing, manuscript preparation

John Egbagba: Protocol writing, sample collection, manuscript preparation

Beleudanyo G. Fente: Sample collection, statistical analysis

All authors read and approved the final manuscript. 


\section{Acknowledgement}

The authors are appreciative to members of staff of the Medical laboratory Science (Microbiology unit) of the Federal Medical Centre, Yenagoa for specimen collection and processing. Authors are also indebted to the participating patients for assenting in this study.

Funding: No funding sources

Conflict of interest: None declared

Ethical Approval: This study was approved by the Institutional Ethics Committee

\section{References}

1. Petrosillo N, Drapeau CM, Nicastri E, Martini L, Ippolito G, Moro ML. Surgical Site Infections in Italian Hospitals: A Prospective Multicenter Study. BMC Infect Dis. 2000;8(1):34. doi: 10.1186/1471-2334-8-34.

2. Patil SB, Paramne A, Harsh S. Antibiotic susceptibility of wound isolates in plastic surgery patients at a tertiary care centre. Indian J Plast Surg. 2016;49(2):198-205. doi:10.4103/0970-0358.191324.

3. Mordi RM, Momoh MI. Incidence of Proteus species in wound infections and their sensitivity pattern in the University of Benin Teaching Hospital. Afri J Biotechnol. 2009;8(5):725-730.

4. Mohammed A, EndrisSeid M, Gebrecherkos T, Tiruneh $\mathrm{M}$, Moges F. Bacterial isolates and their antimicrobial susceptibility patterns of wound infections among inpatient and outpatients attending the University of Gondar referral Hospital, Northwest Ethiopia. Inter J of Microbiol. 2017; 2017:1-10. doi: https://doi.org/10.1155/2017/8953829.

5. Dai T, Huang Y-Y, Sharma SK, Hashmi JT, Kurup DB, Hamblin MR. Topical antimicrobials for burn wound infections. Recent Pat Antiinfect Drug Discov.2010;5 (2):124-151. doi: 10.2174/ 157489110791233522.

6. Vowden P, Cooper RA. Management of wound infection. London: Published by MEP; 2006. An integrated approach to managing wound infections. European wound management association position document; pp. 2-6.

7. Mama M, Abdissa A, Sewunet T. Antimicrobial susceptibility pattern of bacterial isolates from wound infection and their sensitivity to alternative topical agents at Jimma University Specialized Hospital, South-West Ethiopia. Ann Clin Microbiol Antimicrob. 2014; 13:14. doi: 10.1186/1476-0711-13-14.
8. Kotz P, Fisher J, McCluskey P, Hartwell SD, Dharma H: Use of a new silver barrier dressing, Allevyn Ag in exuding chronic wounds. Int Wound J. 2009;6(3):186-194. doi: 10.1111/j.1742-481X.2009. 00608.x.

9. Omoyibo EE, Oladele AO, Ibrahim MH, Adekunle OT. Antibiotic susceptibility of wound swab isolates in a tertiary hospital in Southwest Nigeria. Ann Afr Med. 2018;17 (3):110-116. doi: 10.4103/aam.aam_22_17.

10. Davies J, Davies D. Origins and evolution of antibiotic resistance. Microbiol Mol Biol Rev. 2010;74(3):417-433. doi: 10.1128/MMBR.00016-10.

11. Cooper RA, Molan PC, Harding KG: The sensitivity to honey of Gram-positive cocci of clinical significance isolated from wounds. J App Microbiol. 2002; 93:857-863. doi: 10.1046/j.1365-2672.2002. 01761.x.

12. Pondei K, Fente BG, Oladapo O. Current microbial isolates from wound swabs, their culture and sensitivity pattern at the Niger delta university teaching hospital, Okolobiri, Nigeria. Trop Med Health. 2013;41(2):49-53. doi: 10.2149/tmh.2012-14. Epub 2013 Apr 18.

13. Shittu AO, Kolawole DO, Oyedepo ER. A study of wound infections in two health institutions Ile Ife, Nigeria. Afr. J. BioMed. Res. 2002; 5:97-102.

14. Smith ME, Robinowitz N, Chaulk P, Johnson K. Comparison of chronic wound culture techniques: swab versus curetted tissue for microbial recovery. Br. J. Community Nurs. 2014;(90): S22-S26. doi:10.12968/bjen. 2014.19.Sup9.S22.

15. Clinical and Laboratory Standards Institute (CLSI). Performance Standards for Antimicrobial Susceptibility Testing. Twenty-ninth Information Supplement, 2019; 39(1) M100:1-320.

16. Angel DE, Lloyd P, Carville K, Santamaria N. The clinical efficacy of two semi-quantitative wound-swabbing techniques in identifying the causative organism(s) in infected cutaneous wounds. Int Wound J. 2011;8(2):176185. doi: 10.1111/j.1742-481X.2010.00765.x.

17. Amoran OE, Sogebi AO, Fatugase OM. Rates and risk factors associated with surgical site infections in a tertiary care center in South-Western Nigeria. Int J Trop Dis Health. 2013;26(3):25-36. doi: 10.4103/npmj.npmj_72_19.

18. Ohalete CN, Obi RK, Emeakoroha M C. Bacteriology of different wound infections and their antibiotic 
susceptibility patterns in Imo state, Nigeria. World J Pharm Sci. 2012;1(3):1155-1172.

19. Wu M, Li Y, Guo D, Kui G, Li B, Deng Y, et al. Microbial Diversity of Chronic Wound and Successful Management of Traditional Chinese Medicine. Evid Based Complement Alternat Med. 2018; eCAM,9463295. doi:10.1155/2018/9463295.

20. Basu S, Panray RT, Gulati KA, Singh BT, Shukia KV. A Prospective, Descriptive Study to Identify the Microbiological Profile of Chronic Wounds in Outpatients. Ostomy Wound Manag. 2009;55(1):14-20.

21. Stacy A, McNally L, Darch SE, Brown SP, Whiteley M. The biogeography of polymicrobial infection. Nat Rev

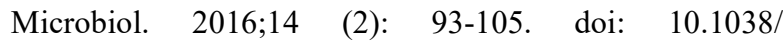
nrmicro.2015.8. Epub 2015.

22. Goswami NN, Trivedi HR, Goswami AP, Patel TK, Tripathi CB. Antibiotic sensitivity profile of bacterial pathogens in postoperative wound infections at a tertiary care hospital in Gujarat, India. J Pharmacol Pharmacother. 2011;2(3):158-164. doi:10.4103/0976-500X.83279.

23. Surucuoglu S, Gazi H, Kurutepe S, Ozkutuk N, Ozbakkaloglu B. Bacteriology of surgical wound infections in a tertiary care hospital in Turkey. East Afr Med J. 2005;82(7):331-336.

24. Janssen H, Janssen I, Cooper P, Kainyah C, Pellio T, Quintel M, et al. Antimicrobial resistant bacteria in infected wounds, Ghana, 2014. Emerg Infect Dis. 2018;24(5):916919. doi: https://doi.org/10.3201/eid2405.171506.

25. Hubab M, Ullah O, Hayat A, Ur Rehman M, Sultana N. Antibiotic susceptibility profile of bacterial isolates from post-surgical wounds of patients in tertiary care hospitals of Peshawar, Pakistan. J Pak Med Assoc. 2018;68(10):15171520 .

26. Srivastava SP, Atal PR, Singh RP. Studies on hospital infections. Indian J Surg. 2015;1969:612-613.

27. Bowler PG, Duerden BI, Amstrong DG. Wound Microbiology and Associated Approaches to Wound Management. Clin Microbiol Rev. 2001;14(2): 244-269. doi: 10.1128/CMR.14.2.244-269.2001.

28. Howell-Jones RS, Wilson MJ, Hill KE, Howard AJ, Price PE, Thomas DW. A review of the microbiology, antibiotic usage and resistance in chronic skin wounds, J Antimicrob Chemother. 2005;55(2):143-149. doi: doi.org/10.1093/jac/dkh513.
29. Gajdács M, Spengler G, Urbán E. Identification and Antimicrobial Susceptibility Testing of Anaerobic Bacteria: Rubik's Cube of Clinical Microbiology? Antibiotics (Basel). 2017;6 (4): E25. doi:10.3390/ antibiotics6040025.

30. Ananth-Shenoy P, Vishwanath S, Targain R, Shetty S, Sunil-Rodrigues G, Mukhopadhyay C. et al. Anaerobic infections in surgical wards: a two-year study. Iran J Microbiol. 2016;8(3):181-186.

31. Brook I., Wexler H M, Goldstein EJ. Antianaerobic antimicrobials: spectrum and susceptibility testing. Clin Microbiol Rev. 2013; 6(3) :526-546. doi:10.1128/ CMR.00086-12.

32. Izadpanah $M$, Khalili $H$. Antibiotic regimens for treatment of infections due to multidrug-resistant Gramnegative pathogens: An evidence-based literature review. J Res Pharm Pract. 2015;4(3):105-114. doi: 10.4103/2279042X.162360.

33. Gorbach S.L. Antibiotic Treatment of Anaerobic Infections, Clin Infect Dis. 1994;18(4):S305-S310. doi: https://doi.org/10.1093/clinids/18.Supplement_4.S305.

34. Blanchette $\mathrm{K} \mathrm{A}$, Wenke JC. Current therapies in treatment and prevention of fracture wound biofilms: why a multifaceted approach is essential for resolving persistent infections. J Bone Jt Infect. 2018;3(2):50-67. doi:10.7150/ jbji.23423.

35. Pfalzgraff A, Brandenburg K, Weindl G. Antimicrobial Peptides and Their Therapeutic Potential for Bacterial Skin Infections and Wounds. Front Pharmacol.2018;9:281. doi: 10.3389/fphar.2018.00281. eCollection 2018 .

36. Raveendran NA, Mohandas A, Menon RR, Menon AS, Biswas R, Jayakumar A. Ciprofloxacin- and FluconazoleContaining Fibrin-Nanoparticle-Incorporated Chitosan Bandages for the Treatment of Polymicrobial Wound Infections. ACS Applied Biomaterials. 2019;2(1):243-254. doi: 10.1021/acsabm.8b00585.

37. Verma P, Chandrakar V, Chitra. Antibiotic sensitivity treatment from Gram-negative bacteria isolated from Pus sample. Inter J Pharm Biol Sci. 2012;2(3):359-363.

38. Vigil KJ, Johnson JR, Johnston BD, Johnston, DP, Kontoyiannis VE, Victor EM, et al. Escherichia coli Pyomyositis: an emerging infectious disease among patients with hematologic malignancies. Clin Infect Dis. 2010;50(3):374-380. doi: 10.1086/649866. 
39. Misra RN, Chander Y, Debata N K, Ohri VC. Antibiotic resistance pattern of isolates from wound and soft tissue infections. Med J Armed Forces India. 2000;56(3):205-208. doi:10.1016/S0377-1237(17)30167-3.

40. Rijal BP, Satyal D, Parajuli N P. High Burden of Antimicrobial Resistance among Bacteria Causing Pyogenic Wound Infections at a Tertiary Care Hospital in Kathmandu, Nepal J Pathog. 2017;9458218:7. doi: https://doi.org/10.1155/2017/9458218.

41. Moremi N, Claus H, Mshana SE. Antimicrobial resistance pattern: a report of microbiological cultures at a tertiary hospital in Tanzania. BMC Infect Dis. 2016;16:756. doi:10.1186/s12879-016-2082-1.

42. Farrag HA, El-Rehim HA, Hazaa MM, Sobhy El-Sayed, SA. Prevalence of Pathogenic Bacterial Isolates Infecting Wounds and their Antibiotic Sensitivity. J Infec Dis Treat. 2016;2:2

43. Shakti L, Veeraraghavan B. Advantage and limitations of nitrofurantoin in multi-drug resistant. Indian scenario. Indian J Med Microbiol. 2015;33(4):477-478. doi: 10.4103/0255-0857.167350.

44. Rego LL, Glazer SC, Zimmern E P. Risks of long-term use of nitrofurantoin for urinary tract prophylaxis in the older patient. Urol. Sci. 2016; 27(4):193-198. doi: https://doi.org/10.1016/j.urols.2016.07.004.

45. Krause KM, Serio A W, Kane TR, Connolly LE. Aminoglycosides: An Overview. Cold Spring Harb Perspect Med. 2016;6(6) :a027029. doi:10.1101/ cshperspect. a027029.

46. Ramirez MS, Tolmasky ME. Aminoglycoside modifying enzymes. Drug Resist Updat. 2010;13(6):151171. doi: 10.1016/j.drup.2010.08.003. Epub 2010 Sep 15.

47. Brook I. Antimicrobial treatment of anaerobic infections. Expert Opin Pharmacother.2011;12(11):16911707. doi: 10.1517/14656566.2011.576672.
48. Nagy E. Anaerobic Infections Update on Treatment Considerations. Drugs. 2010;70(7):841-858. doi: 10.2165/11534490-000000000-00000.

49. Hecker MT, Aron DC, Patel NP, Lehann MK, Donskey CJ. Unnecessary use of antimicrobials in hospitalized patients: Current patterns of misuse with an emphasis on the antianaerobic spectrum of activity. Arch Intern Med. 2003;163(8):972-978. doi: 10.1001/archinte.163.8.972.

50. Sani RA., Garba SA, Oyewole OA. Antibiotic Resistance Profile of Gram-Negative Bacteria Isolated from Surgical Wounds in Minna, Bida, Kontagora and Suleja Areas of Niger State. Am J Med Med Sci. 2012;2(1):20-24. doi: 10.5923/j.ajmms.20120201.05.

51. Hefferman H, Woodhouse R. Antimicrobial Resistance among Gram-Negative Bacilli from Bacteraemia. Antibiotic Reference Laboratory Communicable Disease Group Institute of Environmental Science and Research Ltd. (ESR) 2007; Kenepuru Science Centre.

52. Merchan C, Parajuli S, Siegfried J, Scipione MR, Dubrovskaya Y, Rahimian J. Multidrug-Resistant Bacteroides fragilis Bacteremia in a US Resident: An Emerging Challenge. Case reports in infectious diseases. 2016;2016: 3607125. doi:10.1155/2016/360712.

53. Boyanova L, Kolarov R, Mitov I. Recent evolution of antibiotic resistance in the anaerobes as compared to previous decades. Anaerobe. 2015; 31: 4-10. doi: 10.1016/j.anaerobe.2014.05.004.

54. Waqar A.J., Sajjad MK, Muhammad J, Muazzam M. Surgical site infection and pattern of antibiotic use in a tertiary care hospital in Peshawar. J Ayub Med Coll Abbottabad. 2010;22(3):141-145.

55. Bahashwan SA, El Shafey HM. Antimicrobial resistance patterns of Proteus isolates from clinical specimens. Eur Sci J. 2013;9(27):188-202.

\section{How to cite this article?}

Abdu A.B., Egbagba J., Fente B.G. Identification and antimicrobial susceptibility profile of bacterial pathogens isolated from wound infections in a tertiary hospital, Bayelsa, South southern, Nigeria. Trop J Path Micro 2019;5(12):966-975.doi:10.17511/ jopm.2019.i12.01 